\title{
Retrospective study of irinotecan/cisplatin followed by etoposide/cisplatin or the reverse sequence in extensive-stage small cell lung cancer
}

This article was published in the following Dove Press journal:

OncoTargets and Therapy

2I August 2015

Number of times this article has been viewed

\author{
Xiaoguang Xiao \\ Shujing Wang \\ Shu Xia \\ Man Zou \\ Yang Li \\ Yao Wei \\ Qi Mei \\ Yuan Chen
}

Department of Oncology, Tongji Hospital, Tongji Medical College of Huazhong University of Science and Technology, Wuhan, People's Republic of China
Correspondence: Yuan Chen Department of Oncology, Tongji Hospital, Tongji Medical College of Huazhong University of Science and Technology, 1095 Jiefang Road, Wuhan 430030, People's Republic of China Tel +8602783663407

Email chenyuan008@I63.com
Background: Much research has confirmed the favorable effect of irinotecan/cisplatin (IP) and etoposide/cisplatin (EP) on extensive-stage small cell lung cancer (E-SCLC). This study investigated two sequential orders of IP and EP in the treatment of E-SCLC. We also compared the efficacy and safety of IP and EP in first-line chemotherapy in E-SCLC.

Methods: Ninety-three untreated patients with E-SCLC were randomly allocated to two groups. Group A received IP as first-line therapy until progression and then changed to EP; group B received EP as first-line therapy until tumor progression followed by IP. The primary endpoints were overall survival and time to second tumor progression. The secondary endpoints were first progression-free survival (PFS), ie, time from randomization to first occurrence of tumor progression after first-line treatment with IP or EP, tumor response, and safety of the different sequential treatment orders of IP and EP.

Results: Median overall survival was 15.4 months in group A (IP followed by EP) versus 15.7 months in group B (EP followed by IP; $P=0.483$ ). The median time to second tumor progression was 9.5 months in group A versus 9.9 months in group B $(P=0.361)$. As first-line and second-line therapy, IP achieved a $95.9 \%$ and $60 \%$ disease control rate, respectively, and EP achieved $95.6 \%$ and $59 \%$ disease control rate. The median first PFS was not significantly different between group A and group B (6.5 months and 6.3 months, respectively; $P=0.256)$. Grade 3/4 diarrhea appeared to be significantly more frequent with IP than with EP. The probability of anemia and thrombocytopenia was not significantly different between the two groups. However, significantly more patients who received the IP regimen as second-line treatment developed grade $3 / 4$ neutropenia than those who received the IP regimen as first-line therapy.

Conclusion: There were no statistically significant differences in between the two sequences of IP and EP in the treatment of E-SCLC. Except EP regimen, IP may be another reserved regimen in the first-line treatment of E-SCLC.

Keywords: small cell lung cancer, sequential chemotherapy, irinotecan, etoposide

\section{Introduction}

Small cell lung cancer (SCLC) accounts for nearly $15 \%$ of new lung cancer cases and for about $25 \%$ of lung cancer deaths annually. ${ }^{1}$ Although the efficacy of chemotherapy for SCLC may be as high as $80 \%$, the 1-year and 2-year survival rates are only $35 \%-45 \%$ and $10 \%-20 \%$, respectively. ${ }^{2}$ The long-term prognosis is very poor. Chemotherapy plays a key role in the treatment of extensive-stage SCLC (E-SCLC), in that it not only alleviates symptoms but also prolongs survival in most patients. ${ }^{3,4}$ Etoposide and cisplatin (EP) is a classic chemotherapy regimen and is very widely used. ${ }^{5,6}$ The traditional standard treatment for E-SCLC is etoposide and cisplatin, and this combination alternating with a regimen of cyclophosphamide, doxorubicin, and 
vincristine yields a median survival of 8-10 months and a 2 -year survival rate of $10 \%$. Some newer agents, including the taxanes, vinorelbine, gemcitabine, topotecan, and irinotecan have shown significant activity in single use. However, in the last decade, a large number of platinumbased combination therapies tested in Phase III trials failed to demonstrate efficacy superior to that of EP, until the advent of irinotecan. ${ }^{7}$ In 2000, the Japan Clinical Oncology Group reported a randomized clinical trial (JCOG-9511) of 154 patients with E-SCLC treated with irinotecan in combination with cisplatin (IP) or EP as first-line treatment. Median overall survival (OS) was significantly longer in the IP group than in the EP group (12.8 months and 9.4 months, respectively, $P<0.002){ }^{8}$ This was the first trial in over 20 years to demonstrate a significant improvement in survival using a regimen other than EP. Hermes et al subsequently reported that IP not only improved quality of life scores but also prolonged survival in patients with E-SCLC, ${ }^{9}$ and a further clinical trial demonstrated that IP could improve PFS compared with less toxicities in E-SCLC. ${ }^{10,11}$ Therefore, the National Comprehensive Cancer Network guidelines added the IP protocol as a first-line therapy for patients with E-SCLC. However, two subsequent randomized studies conducted in the USA, Canada, and Australia failed to demonstrate a difference in OS between their IP and EP treatment arms, so the role of the IP regimen remains controversial in patients with E-SCLC. ${ }^{12}$

Irinotecan and etoposide have demonstrated improvement in survival when combined with cisplatin in first-line and second-line therapy. As we have discussed above, these two drugs may be only effective but difficult to choose in clinical. EP and IP are both effective regimens in the treatment of SCLC, but limited information is available concerning their optimal sequential order. In this retrospective study, we compared the efficacy and safety of IP and EP when used as first-line therapy in E-SCLC, and given the tendency of SCLC to relapse, we also attempted to identify the optimal sequence of IP and EP when treating patients with the disease.

\section{Methods and materials Patient characteristics}

In this study, follow-up data were analyzed for 93 patients treated for E-SCLC in the oncology department at Tongji Hospital between January 2011 and November 2013. Histopathological specimens was obtained by fiberoptic bronchoscopy, computed tomography (CT)-guided transthoracic needle aspiration, or cervical lymph node biopsy. The pathological diagnosis of SCLC was reviewed and confirmed by two separate pathologists using the Veterans Administration Lung Study Group staging system to identify E-SCLC.

The study inclusion criteria were Eastern Cooperative Oncology Group score 0 to 2; essentially normal routine blood, liver and kidney function tests before treatment; assessable disease; and written informed consent. The patients were assigned to receive IP until progression and then EP (group A) or the reverse treatment sequence (group B, Table 1).

The study was approved by the medical ethics committee of Tongji Hospital, Tongji Medical College of Huazhong University of Science and Technology. All patients enrolled in this study provided written informed consent.

\section{Chemotherapy}

IP consisted of irinotecan $\left(60 \mathrm{mg} / \mathrm{m}^{2}\right.$ intravenously on days 1,8 , and 15$)$ and cisplatin $\left(75 \mathrm{mg} / \mathrm{m}^{2}\right.$ intravenously on days 1$)$ every 4 weeks. EP consisted of etoposide $\left(100 \mathrm{mg} / \mathrm{m}^{2}\right.$ on days $1-3)$ and cisplatin $\left(75 \mathrm{mg} / \mathrm{m}^{2}\right.$ intravenously on

Table I Clinical characteristics of the two groups

\begin{tabular}{|c|c|c|c|}
\hline Item & Group A n (\%) & Group B n (\%) & $P$-value \\
\hline Number & 48 & 45 & \\
\hline Sex & & & 0.392 \\
\hline Male & $30(62.5)$ & $28(62.2)$ & \\
\hline Female & $18(37.5)$ & $17(37.8)$ & \\
\hline \multicolumn{4}{|l|}{ Age (years) } \\
\hline Median value & 56 & 57 & 0.516 \\
\hline Range & $31-68$ & $34-70$ & \\
\hline \multicolumn{4}{|l|}{ ECOG score } \\
\hline Median value & 1 & I & \\
\hline \multicolumn{4}{|l|}{ Smoking } \\
\hline Yes & $28(58.3)$ & $25(55.5)$ & 0.417 \\
\hline No & $20(4 \mid .7)$ & $20(44.5)$ & \\
\hline \multicolumn{4}{|l|}{ LDH } \\
\hline$\leq 225 \mathrm{IU} / \mathrm{L}$ & $19(39.6)$ & $18(40.0)$ & 0.562 \\
\hline$>225 \mathrm{IU} / \mathrm{L}$ & $29(60.4)$ & $27(60.0)$ & \\
\hline \multicolumn{4}{|l|}{ NSE } \\
\hline$\leq 16 \mu \mathrm{g} / \mathrm{L}$ & $6(1.3)$ & $4(0.9)$ & 0.543 \\
\hline$>16 \mu g / L$ & $42(97.7)$ & $41(99.1)$ & \\
\hline \multicolumn{4}{|l|}{ SIADH } \\
\hline Yes & $39(8 \mathrm{I} .3)$ & $38(84.4)$ & 0.879 \\
\hline No & $9(18.7)$ & $7(15.6)$ & \\
\hline \multicolumn{4}{|c|}{ Chemotherapy cycle first-line } \\
\hline Median value & 5 & 5 & \\
\hline \multicolumn{4}{|l|}{ Metastatic sites } \\
\hline Liver & $12(25.0)$ & $10(22.2)$ & 0.731 \\
\hline Bone & $10(20.8)$ & $8(17.8)$ & 0.582 \\
\hline Brain & 20 (4I.7) & $18(40.0)$ & 0.696 \\
\hline Other & $6(12.5)$ & $9(20.0)$ & 0.542 \\
\hline
\end{tabular}

Note: Patients received IP until progression and then EP (group A), or the reverse treatment sequence (group B).

Abbreviations: ECOG, Eastern Cooperative Oncology Group; LDH, lactic dehydrogenase; NSE, neuron-specific enolase; SIADH, syndrome of inappropriate secretion of antidiuretic hormone. 
day 1) every 3 weeks. Antiemetic prophylaxis with a 5-hydroxytryptamine 3-receptor antagonist was administered routinely. The dose of cisplatin could be divided into three days use depending on the patient's performance status and their ability to tolerate chemotherapy. The doses of irinotecan, etoposide, and cisplatin could be adjusted according to the severity of adverse events.

\section{Follow-up visits}

A physical examination, full blood count, and hepatic and renal function tests were performed before each cycle of chemotherapy. Measurable lesions were evaluated at baseline by CT scan. A CT scan was repeated for every two cycles of chemotherapy or adjusted according to illness.

After 3 months of systemic therapy, each patient underwent a contrast-enhanced CT scan of the chest or an ultrasound B mode or CT scan of the upper abdomen. All patients were provided with information on smoking cessation at the time of diagnosis. Follow-up was performed in hospital, in the outpatient clinic, and by telephone or email. Dates of first and second occurrence of tumor progression were recorded.

\section{Study endpoints}

The primary study endpoints were OS and the time to second tumor progression (TTsP). OS was defined as the interval between randomization and death from any cause and TTsP was defined as the interval between randomization and second relapse. The secondary study endpoints were first progression-free survival (PFS, ie, the interval between randomization and first occurrence of tumor progression after first-line treatment with IP or EP), tumor response, and safety. The Response Evaluation Criteria in Solid Tumors criteria were used to evaluate the tumor response. Adverse events were evaluated using National Cancer Institute Common Toxicity Criteria-Adverse Effect version 3.0.

\section{Statistical analysis}

The data were analyzed using the Statistical Package for the Social Sciences version 18.0 software (SPSS Inc, Chicago, IL, USA). The survival curves, PFS curves, and TTsP were evaluated by the Kaplan-Meier method, and the groups were compared using the log-rank test. $P<0.05$ was considered to indicate a statistically significant difference.

\section{Results \\ Overall survival}

Median OS was 15.4 months (95\% confidence interval [CI] 13.9-16.9) in group A and 15.7 months (95\% CI 14.0-17.5) in group B; the difference between the two groups was not statistically significant $(P=0.483$, Figure 1$)$.

\section{Time to second tumor progression}

The median TTsP was 9.5 months (95\% CI 8.6-10.1) in group A and 9.9 months (95\% CI 8.8-10.9) in group B. Significant difference was also observed between the two groups $(P=0.361$; Figure 2$)$.

\section{First progression-free survival}

The median duration of first PFS was 6.5 months $(95 \%$ CI 5.7-7.2) in group A and 6.3 months (95\% CI 5.5-7.2) in group $\mathrm{B}$; this difference was not statistically significant between the two groups ( $P=0.256$; Figure 3$)$. The median delay between first PFS and the start of second-line chemotherapy was 21 days in group A and 15 days in group B.

\section{Objective tumor response}

In first-line therapy, nine complete responses were achieved with IP (18.8\%) versus eight complete responses with EP (17.8\%). The disease control rates were $95.9 \%$ with IP versus $95.6 \%$ with $\operatorname{EP}(P=0.487)$. All patients enrolled in the study accepted second-line chemotherapy. Three complete responses were observed with EP (6.2\%) and two complete responses with IP (4.4\%). The disease control rates were $60 \%$ with IP and 59\% with EP ( $P=0.514$; Table 2$)$.

\section{Adverse events}

There was one chemotherapy-related death in group A due to life-threatening hematological toxicity and delayed diarrhea. Grade 3/4 hematological toxicity was significantly more frequent with EP, and grade 3/4 diarrhea was significantly more frequent with IP. The frequency of adverse effects during first-line and second-line treatment in each group are summarized in Table 3. For the EP regimen, the incidence of grade 3/4 neutropenia in first-line treatment was significantly higher

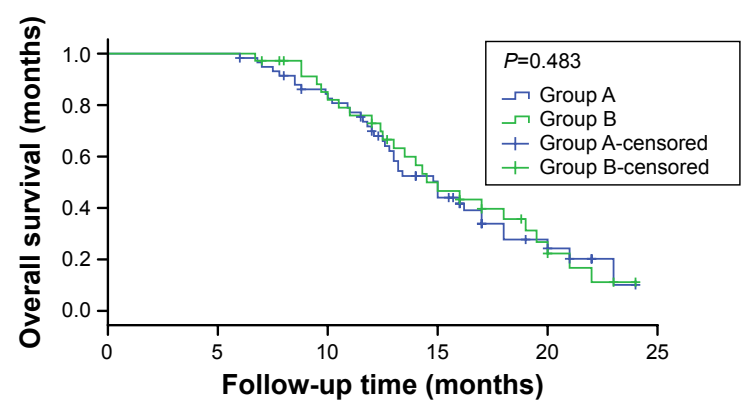

Figure I Overall survival in the two groups.

Note: Patients received IP until progression and then EP (group A), or the reverse treatment sequence (group B).

Abbreviations: IP, irinotecan/cisplatin; EP, etoposide/cisplatin. 


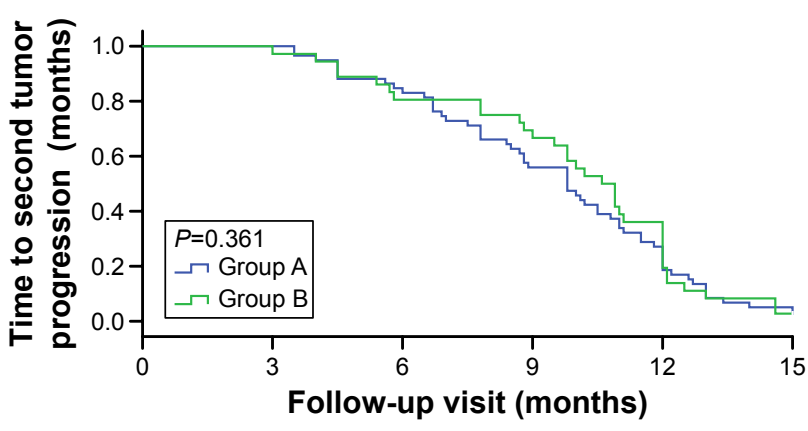

Figure 2 Time to second tumor progression in the two groups.

Note: Patients received IP until progression and then EP (group A), or the reverse treatment sequence (group B).

Abbreviations: IP, irinotecan/cisplatin; EP, etoposide/cisplatin.

than that with IP, and the incidence of grade 3/4 diarrhea was significantly lower than that of IP. For the IP regimen, the incidence of grade 3/4 neutropenia was increased by second-line treatment, and diarrhea and thrombocytopenia had no difference between groups. The probability of anemia and thrombocytopenia was not statistically significant in the first-line and second-line treatment between two groups. In the two treatment groups, the patients accepted IP regimen as second-line treatment occurred $3 / 4$ grade neutropenia was significantly higher than that of IP regimen as first-line therapy. At the same time, for the patients received the EP regimen as the first-line and second-line treatment, the incidence of grade $3 / 4$ hematological and non-hematological toxicity changed little.

\section{Discussion}

SCLC has a much worse biological behavior than non-SCLC, and the disease develops and metastasizes rapidly. Although SCLC is highly sensitive to radiotherapy and chemotherapy, the maintenance time is short, and most patients die within 2 years. About $70 \%-80 \%$ of patients with limited disease and almost all of those with extensive-stage disease develop progression or recurrence after several months. The original chemotherapy regimen also demonstrated ineffective. The response to second-line chemotherapy is highly relevant to the time from the beginning to the recurrence time after the

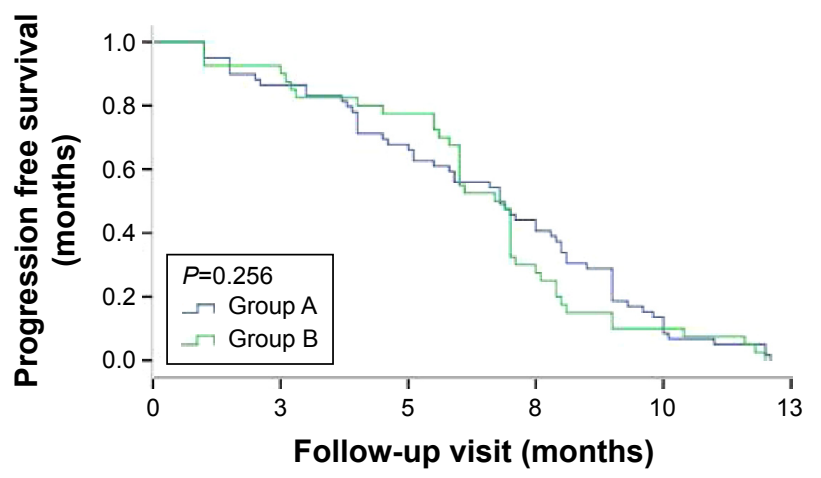

Figure 3 First overall progression-free survival duration in the two groups. Note: Patients received IP until progression and then EP (group A), or the reverse treatment sequence (group $B$ ).

Abbreviations: IP, irinotecan/cisplatin; EP, etoposide/cisplatin.

first-line chemotherapy; the second-line treatment response rate is often less than $10 \%$ if this is less than 3 months, and often higher than $25 \%$ if it is more than 3 months. However, the evidence base concerning the value of second-line chemotherapy for relapsed SCLC is limited, and the role of targeted therapy in SCLC has not been confirmed. Therefore, there is an urgent need to identify an effective treatment for patients with refractory SCLC but otherwise good performance status.

The EP regimen is the first-line chemotherapy most commonly used for E-SCLC. The response rate for EP is $60 \%-80 \%$, and the median patient survival was 8-10 months. Irinotecan is one of the S-phase cell cyclespecific drugs. It is metabolized to its active form, 7-ethyl10-hydroxycamptothecin ( $\mathrm{SN}-38)$, by carboxylesterase in the liver. SN-38 then exerts its cytotoxic effect by inhibiting topoisomerase I, which is required for DNA replication, thereby damaging single-stranded DNA and blocking DNA replication, resulting in inhibition of cell division and death of tumor cells. ${ }^{13}$ The IP protocol of irinotecan and platinum drugs has been explored in SCLC. JCOG-9511, a Phase III trial of IP and EP in E-SCLC patients, showed that IP significantly prolonged survival compared with EP (12.8 months versus 9.4 months, $P=0.002$ ) in a first time. ${ }^{8}$ This trial was terminated early because the IP group showed significant survival and safety benefits in the interim analysis. Schmittel et al

Table 2 Short-term therapeutic effect of the two sequences

\begin{tabular}{|c|c|c|c|c|c|c|}
\hline & \multicolumn{3}{|l|}{ First-line } & \multicolumn{3}{|l|}{ Second-line } \\
\hline & Group A IP & Group B EP & $P$-value & Group A EP & Group B IP & $P$-value \\
\hline CR & 9 & 8 & 0.92 & 3 & 2 & 0.45 \\
\hline PR & 31 & 28 & 0.63 & 14 & 10 & 0.66 \\
\hline SD & 6 & 7 & 0.47 & 12 & 15 & 0.24 \\
\hline PD & 2 & 2 & 0.28 & 20 & 18 & 0.52 \\
\hline
\end{tabular}

Note: Patients received IP until progression and then EP (group A, $n=48$ ), or the reverse treatment sequence (group $B, n=45)$.

Abbreviations: IP, irinotecan/cisplatin; EP, etoposide/cisplatin; CR, complete response; PR, partial response; SD, stable disease; PD, progressive disease. 
Table 3 Adverse events according to sequential order of treatment

\begin{tabular}{|c|c|c|c|c|c|c|}
\hline & \multicolumn{3}{|l|}{ First-line } & \multicolumn{3}{|l|}{ Second-line } \\
\hline & Group A IP & Group B EP & $P$-value & Group B IP & Group A EP & $P$-value \\
\hline Anemia & 2 & 5 & 0.249 & 6 & 9 & 0.195 \\
\hline Neutropenia & 11 & 23 & 0.015 & 22 & 26 & 0.486 \\
\hline Thrombocytopenia & 9 & 7 & 0.316 & 12 & 9 & 0.238 \\
\hline Diarrhea & 10 & 2 & 0.012 & 11 & 4 & 0.017 \\
\hline
\end{tabular}

Note: Patients received IP until progression and then EP (group A), or the reverse treatment sequence (group B).

Abbreviations: IP, irinotecan/cisplatin; EP, etoposide/cisplatin.

and Hermes et al also reported that the IP scheme can improve OS and PFS in patients with E-SCLC. ${ }^{910}$ However, subsequent major studies conducted to confirm the superiority of IP did not yield positive results. ${ }^{14,15}$ In addition, a recent meta-analysis found that OS may be better with IP than with EP. ${ }^{7}$ Differences in treatment outcomes between Asian and Western populations and the small patient population included were the main explanations put forward for the failure to observe a survival difference in JCOG-9511.

As a special pathological type, progress in the treatment of SCLC has been slow in the past few decades. ${ }^{16}$ With the exception of IP and EP, emerging chemotherapy drugs, including targeted therapy and immune therapy, have not lived up to their initial promise in the treatment of SCLC. ${ }^{17,18}$

Although IP and EP are both effective chemotherapy regimens for E-SCLC, there has been little research on the advantages and disadvantages of the sequential order of these therapies. EP and IP scheme in sequential order in the study of E-SCLC show that the two different sequential orders all showed better curative effect. The median survival time for the two groups was 15.4 months and 15.7 months, respectively, ie, higher than in JCOG-9511, and also higher than in the large clinical studies in the USA, Australia, and Canada, suggesting that EP and IP scheme of sequential treatment with more survival benefit. More importantly, the sequence of IP followed by EP, or the reverse order of EP followed by IP, failed to prolong survival. The short-term and long-term effects of the two groups were not significantly different, but the regimen with the least toxicity is preferred. Given some patients may not receive second-line treatment for physical or economic reasons, first-line chemotherapy is particularly important, and subsequent therapy after tumor progression may greatly influence OS. ${ }^{19}$

However, what is worth emphasizing is the adaptability of patients. For the patient first receiving the IP treatment in group A, severe lethality adverse, such as diarrhea, may make the patient fear the procedure. Intractable diarrhea may be very difficult to treat, which is demoralizing for the patient, and may adversely impact follow-up treatment method. Hematological toxicity may be easily managed using G-CSF or GM-CSF, enabling effective implementation of the treatment plan. This may explain why OS in group B was a little longer than in group A in our present study. Although no significant difference was found between the two groups, we still pay more attention to the little difference. Uridine diphosphate glucuronosyltransferase (UGT)1A1 has played an important role in the metabolism of irinotecan. Extensive research attention had paid to the correlation between UGT1A1 gene polymorphisms and irinotecan-related delayed diarrhea and neutropenia, and since 2005 the US Food and Drug Administration has required that testing for UGT1A $1 * 28$ as a risk factor for severe adverse events be included on the irinotecan label. ${ }^{20}$ In routine clinical practice, analysis of UGT1A1 polymorphism should be recommended to guide the use of irinotecan. ${ }^{21}$

Our study found that IP produced less grade $3 / 4$ hematological toxicity but more grade $3 / 4$ diarrhea than EP. The incidence of 3/4 grade neutropenia when using EP as first-line treatment was significantly higher than when using IP (56\% in group $\mathrm{B}$ versus $29 \%$ in group $\mathrm{B}, P=0.015$ ), and the incidence of 3/4 grade diarrhea was significantly lower when using EP than when using IP (33\% in group A versus $16 \%$ in group B, $P=0.012) ; 3 / 4$ grade neutropenia occurred significantly more often in patients receiving IP as second-line treatment than in those receiving IP as first-line therapy, whereas the incidence of 3/4 grade hematological and non-hematological toxicity was similar in those receiving EP as first-line or second-line treatment. Although the overall PFS was similar between the two groups, group A showed a slight advantage compared with group B. Therefore, the IP regimen may be an alternative to the EP regimen in first-line treatment of E-SCLC. In terms of side effects, it is suggested that the toxicity of IP as a first-line treatment is less, and that a protocol of IP followed by EP is the preferred sequential program.

In summary, the short-term and long-term effects of these two sequential treatments for E-SCLC is similar, but the toxicity of IP as first-line treatment is less. However, considering adverse events and patient compliance, irinotecan should be 
used cautiously in patients carrying abnormal UGT1A1 gene polymorphism. But only consider the first-line treatment of E-SCLC IP remains an appropriate choice. Large, randomized, double-blind, prospective studies are still necessary.

\section{Disclosure}

The authors report no conflicts of interest in this work.

\section{References}

1. Govindan R, Page N, Morgensztern D, et al. Changing epidemiology of small-cell lung cancer in the United States over the last 30 years: analysis of the surveillance, epidemiologic, and end results database. J Clin Oncol. 2006;24(28):4539-4544.

2. Xiao XG, Chen Y, Wang SJ, et al. Relationship between the SER treatment period and prognosis of patients with small cell lung cancer. Asian Pac J Cancer Prev. 2014;15(15):6415-6419.

3. Kim HG, Lee GW, Kang JH, et al. Combination chemotherapy with irinotecan and cisplatin in elderly patients ( $\geq 65$ years) with extensivedisease small-cell lung cancer. Lung Cancer. 2008;61(2):220-226.

4. Chen G, Huynh M, Fehrenbacher L, et al. Phase II trial of irinotecan and carboplatin for extensive or relapsed small cell lung cancer. J Clin Oncol. 2008;27(9):1401-1404.

5. Ettinger DS. New drugs for chemotherapy-naive patients with extensive disease small cell lung cancer. Semin Oncol. 2001;28(2):27-29.

6. Luo J, Wu FY, Li AW, et al. Comparison of vinorelbine, ifosfamide and cisplatin (NIP) and etoposide and cisplatin (EP) for treatment of advanced combined small cell lung cancer (cSCLC) patients: a retrospective study. Asian Pac J Cancer Prev. 2012;13(9):4703-4706.

7. Jiang L, Yang KH, Guan QL, et al. Cisplatin plus etoposide versus other platin-based regimens for patients with extensive small-cell lung cancer: a systematic review and meta-analysis of randomised, controlled trials. Intern Med J. 2012;42(12):1297-1309.

8. Noda K, Nishiwaki Y, Kawahara M, et al. Irinotecan plus cisplatin compared with etoposide plus cisplatin for extensive small-cell lung cancer. N Engl J Med. 2002;346(2):85-91.

9. Hermes A, Bergman B, Bremnes R, et al. Irinotecan plus carboplatin versus oral etoposide plus carboplatin in extensive small-cell lung cancer: a randomized phase III trial. J Clin Oncol. 2008;26(26):4261-4267.
10. Schmittel A, Fischer von Weikersthal L, Sebastian M, et al. A randomized phase II trial of irinotecan plus carboplatin versus etoposide plus carboplatin treatment in patients with extended disease small-cell lung cancer. Ann Oncol. 2006;17(4):663-667.

11. Schmittel A, Sebastian M, Fischer von Weikersthal L, et al. A German multicenter, randomized phase III trial comparing irinotecan-carboplatin with etoposide-carboplatin as first-line therapy for extensive-disease small-cell lung cancer. Ann Oncol. 2011;22(8):1798-1804.

12. Jiang J, Liang X, Zhou X, et al. A meta-analysis of randomized controlled trials comparing irinotecan/platinum with etoposide/platinum in patients with previously untreated extensive-stage small cell lung cancer. J Thorac Oncol. 2010;5(6):867-873.

13. Di Paolo A, Bocci G, Polillo M, et al. Pharmacokinetic and pharmacogenetic predictive markers of irinotecan activity and toxicity. Curr Drug Metab. 2011;12(10):932-943.

14. Lara PN Jr, Natale R, Crowley J, et al. Phase III trial of irinotecan/ cisplatin compared with etoposide/cisplatin in extensive-stage smallcell lung cancer: clinical and pharmacogenomic results from SWOG S0124. J Clin Oncol. 2009;27(15):2530-2535.

15. Zatloukal P, Cardenal F, Szczesna A, et al. A multicenter international randomized phase III study comparing cisplatin in combination with irinotecan or etoposide in previously untreated small-cell lung cancer patients with extensive disease. Ann Oncol. 2010;21(9):1810-1816.

16. Kalemkerian GP. Advances pharmacotherapy of small-cell lung cancer. Expert Opin Pharmacother. 2014;15(16):2385-2396.

17. Pillai RN, Owonikoko TK. Small cell lung cancer: therapies and targets. Semin Oncol. 2014;41(1):133-142.

18. Ehrlich D, Wang B, Lu W, et al. Intratumoral anti-HuD immunotoxin therapy for small cell lung cancer and neuroblastoma. J Hematol Oncol. 2014;7(1):91.

19. Imai H, Mori K, Wakuda K, et al. Progression-free survival, postprogression survival, and tumor response as surrogate markers for overall survival in patients with extensive small cell lung cancer. Ann Thorac Med. 2015;10(1):61-66.

20. Li M, Wang Z, Guo J, et al. Clinical significance of UGT1A1 gene polymorphisms on irinotecan-based regimens as the treatment in metastatic colorectal cancer. Onco Targets Ther. 2014;23(7):1653-1661.

21. Stewart CF, Panetta JC, OShaughnessy MA, et al. UGT1A1 promoter genotype correlates with SN-38 pharmacokinetics but not severe toxicity in patients receiving low-dose irinotecan. J Clin Oncol. 2007;25(18): 2594-2600.
OncoTargets and Therapy

\section{Publish your work in this journal}

OncoTargets and Therapy is an international, peer-reviewed, open access journal focusing on the pathological basis of all cancers, potential targets for therapy and treatment protocols employed to improve the management of cancer patients. The journal also focuses on the impact of management programs and new therapeutic agents and protocols on

\section{Dovepress}

patient perspectives such as quality of life, adherence and satisfaction. The manuscript management system is completely online and includes a very quick and fair peer-review system, which is all easy to use. Visit http://www.dovepress.com/testimonials.php to read real quotes from published authors. 